\title{
Numerical solutions of the incompressible miscible displacement equations in heterogeneous media
}

\author{
Jizhou Li ${ }^{1}$, Beatrice Riviere ${ }^{1}$ \\ Department of Computational and Applied Mathematics, Rice University, Houston, TX \\ 77005
}

\begin{abstract}
This paper presents a numerical method based on mixed finite element, discontinuous Galerkin methods in space and high order Runge-Kutta method in time for solving the miscible displacement problem. No slope limiters are needed. The proposed method exhibits high order of convergence in space and time when comparing with analytical solutions. The simulation shows robustness of the method for heterogeneous media with highly varying permeabilities.
\end{abstract}

Keywords: High order, mixed finite elements, discontinuous Galerkin, Runge-Kutta methods

\section{Introduction}

$\mathrm{CO}_{2}$ enhanced oil recovery has received a lot of attention from industry, government and environmental organizations for both its potential to increase U.S. oil production and its potential for permanently storing $\mathrm{CO}_{2}$. In this paper, we study the miscible displacement of one fluid by another in a porous medium. The mathematical model is a system of coupled elliptic and convection-dominated parabolic equations. While there is an extensive mathematical analysis for the coupling of elliptic and parabolic equations, there is a need for developing efficient and accurate numerical methods for solving the miscible displacement problem in realistic porous media. One

Email addresses: Jizhou.Li@rice.edu (Jizhou Li), riviere@rice.edu (Beatrice Riviere)

${ }^{1}$ The authors acknowledge the support of NSF by the grant NSF-DMS 1318348 .

Preprint submitted to Computer Methods in Applied Mechanics and EngineeringOctober 28, 2014

(C) 2014. This manuscript version is made available under the Elsevier user license http://www.elsevier.com/open-access/userlicense/1.0/ 
numerical difficulty is the approximation of intricate velocity fields resulting from highly varying permeabilities. Ideal numerical methods should yield negligible artificial diffusion as the accurate front tracking of the fluid mixture has direct economical consequences.

We propose a new numerical method of arbitrary order for solving the incompressible miscible displacement problem in heterogeneous media. The spatial discretization combines the mixed finite element (MFE) method for the pressure and velocity equations, and a modified interior penalty discontinuous Galerkin (DG) method for the concentration equation. The time discretization for the concentration equation is based on a class of implicit Runge Kutta methods with lower triangular Butcher tables. In this computational paper, we show that our method is robust and exhibits very sharp concentration fronts. In addition, the coupling strategy we propose preserves the high order in time convergence rate for smooth solutions. The analysis of our proposed method can be found in [10]. In that theoretical work, we show convergence of the numerical solutions to the weak solution as the mesh size and time step tend to zero. The case of low regularity is handled by our analysis. Our particular choice of Runge-Kutta methods is based on the known relationship between those time-stepping techniques and the discontinuous Galerkin in time method $[11,12]$. In other words, our proposed method is equivalent to a combined mixed finite element, discontinuous Galerkin method in space with a discontinuous Galerkin method in time. It is however computationally more efficient to rewrite the time-stepping method in the Runge-Kutta framework. While the theoretical analysis in [10] guarantees convergence of the method for non-smooth solutions, this current paper shows the numerical behavior of the solution for realistic heterogeneous media.

Various numerical methods have been applied to the miscible displacement problem and their convergence has been obtained for smooth solutions (see for instance $[2,3,4,5,6,7,8]$ ). In $[8,9]$, a first order method in time is combined with standard DG methods in space, and additional stabilization techniques (such as a cut-off operator and slope limiters) are needed. More recently convergence of the methods has been obtained for low-regularity solutions. In [13], a first-order in time (backward Euler) is combined with MFE and DG in space. The treatment of the dispersion-diffusion term in [13] involves a projection onto the space of piecewise constants. This work is extended to a second-order in time (Crank-Nicolson) in [14]. In [15], theoretical convergence is obtained for a high order DG in time scheme combined 
with MFE and continuous finite element methods.

The outline of the paper is as follows. After a brief description of the mathematical problem in Section 2, we introduce the semi-discrete scheme in Section 3. The coupling strategy is defined in Section 4 and numerical simulations are shown in Section 5. Conclusions follow.

\section{Model problem}

The displacement of the single phase fluid mixture in the porous medium $\Omega \subset \Re^{2}$ over a time interval $[0, T]$ is characterized by the following mathematical model:

$$
\begin{aligned}
& \nabla \cdot \mathbf{u}=q^{I}-q^{P}, \quad \text { in } \quad \Omega \times(0, T), \\
& \mathbf{u}=-\mathbf{K}(c)(\nabla p-\rho(c) \mathbf{g}), \quad \text { in } \Omega \times(0, T), \\
& \partial_{t}(\phi c)-\operatorname{div}(\mathbf{D}(\mathbf{u}) \nabla c-c \mathbf{u})=q^{I} \hat{c}-q^{P} c, \quad \text { in } \quad \Omega \times(0, T) \text {, }
\end{aligned}
$$

where the physical unknowns are $p$ the fluid pressure, $\mathbf{u}$ the velocity and $c$ the concentration of the solvent.

The flow and transport processes are driven by the functions $q^{I}$ and $q^{P}$ which represent injection wells and production wells respectively. The other coefficients in the system are the fluid density $\rho(c)$, the gravity vector $\mathbf{g}$, the porosity of the medium $\phi$, the diffusion-dispersion matrix $\mathbf{D}(\mathbf{u})$, the injected concentration $\hat{c}$, and the matrix $\mathbf{K}(c)$, which is the ratio between the permeability matrix $\mathbf{k}$ and the fluid viscosity $\mu(c)$. The initial concentration is denoted by $c_{0}$. We complete the system by no-flow boundary conditions:

$$
\mathbf{u} \cdot \mathbf{n}=0, \quad \text { and } \quad \mathbf{D}(\mathbf{u}) \nabla c \cdot \mathbf{n}=0, \quad \text { on } \quad \partial \Omega \times(0, T),
$$

and by an additional constraint on the pressure for uniqueness.

\section{Semi-discrete scheme}

We introduce the spatial discretization of the model problem. Equations (1), (2) are discretized by the mixed finite element method and equation (3) by an interior penalty discontinuous Galerkin (IPDG) method. Let $\left\{\mathcal{E}_{h}\right\}_{h>0}$ be a regular family of meshes of $\Omega$, where $h$ is the maximum element diameter. The velocity, pressure and concentration are approximated in the 
following finite-dimensional subspaces:

$$
\begin{aligned}
& \mathbf{U}_{h}=\left\{\mathbf{u}_{h} \in H(\Omega ; \operatorname{div})\left|\mathbf{u}_{h}\right|_{E} \in\left(\mathcal{P}_{k}(E)\right)^{d}+x \mathcal{P}_{k}(E), E \in \mathcal{E}_{h}\right\}, \\
& P_{h}=\left\{q_{h} \in L^{2}(\Omega):\left.q_{h}\right|_{E} \in \mathcal{P}_{k}(E), E \in \mathcal{E}_{h}\right\}, \\
& C_{h}=\left\{c_{h} \in L^{2}(\Omega):\left.c_{h}\right|_{E} \in \mathcal{P}_{r}(E), E \in \mathcal{E}_{h}\right\} .
\end{aligned}
$$

Here $\mathcal{P}_{k}(E)$ denotes the space of polynomials of degree less than or equal to $k$ over the element $E$. In our simulations, the Raviart-Thomas spaces are used; however, any classical mixed finite element spaces would be suitable (see for instance [16]).

The numerical solution of the concentration is discontinuous across mesh elements. To define the jump $[\cdot]$ and average $\{\cdot\}$ of a discontinuous function, let $\Gamma_{h}$ denote the set of interior faces. Then for each edge $e$ in $\Gamma_{h}$ fix a normal vector $\mathbf{n}_{e}$ and let $E_{+}^{e}$ and $E_{-}^{e}$ denote the neighboring elements such that $\mathbf{n}_{e}$ points from $E_{+}^{e}$ to $E_{-}^{e}$. Then we define

$$
\{v\}=\frac{\left.v\right|_{E_{+}^{e}}+\left.v\right|_{E_{-}^{e}}}{2}, \quad \text { and } \quad[v]=\left.v\right|_{E_{+}^{e}}-\left.v\right|_{E_{-}^{e}} .
$$

The $L^{2}$ inner-product on $\mathcal{E}_{h}$ and $\Gamma_{h}$ are:

$$
(\cdot, \cdot)_{\mathcal{E}_{h}}=\sum_{E \in \mathcal{E}_{h}}(\cdot, \cdot)_{E}, \quad(\cdot, \cdot)_{\Gamma_{h}}=\sum_{e \in \Gamma_{h}}(\cdot, \cdot)_{e}
$$

We can rewrite Equation (3) in the following form:

$$
\partial_{t}(\phi c)-\operatorname{div}(\mathbf{D}(\mathbf{u}) \nabla c)+\frac{1}{2}\left(\operatorname{div}(c \mathbf{u})+\mathbf{u} \cdot \nabla c+\left(q^{I}+q^{P}\right) c\right)=q^{I} \hat{c} .
$$

We then apply the IPDG method and denote by $B_{d}(\cdot, \cdot ; \cdot)$ the IPDG discretization of the operator $-\operatorname{div}(\mathbf{D}(\mathbf{u}) \nabla c)$ with penalty coefficient, $\sigma>0$, and parameter $\epsilon \in\{-1,0,1\}$ :

$$
\begin{aligned}
B_{d}\left(c_{h}, w_{h} ; \mathbf{u}_{h}\right)=\left(\mathbf{D}\left(\mathbf{u}_{h}\right) \nabla c_{h}, \nabla w_{h}\right)_{\mathcal{E}_{h}}-\left(\left[w_{h}\right],\left\{\mathbf{D}\left(\mathbf{u}_{h}\right) \nabla c_{h} \cdot \mathbf{n}_{e}\right\}\right)_{\Gamma_{h}} \\
+\epsilon\left(\left[c_{h}\right],\left\{\mathbf{D}\left(\mathbf{u}_{h}\right) \nabla w_{h} \cdot \mathbf{n}_{e}\right\}\right)_{\Gamma_{h}}+\left(\sigma h^{-1}\left(1+\left\{\left|\mathbf{u}_{h}\right|\right\}\right)\left[c_{h}\right],\left[w_{h}\right]\right)_{\Gamma_{h}} .
\end{aligned}
$$

We note that the weight $1+\left\{\left|\mathbf{u}_{h}\right|\right\}$ in the penalty term is needed for the analysis of the method in the general case of unbounded diffusion-dispersion matrix $[10]$. 
The form $B_{c q}(\cdot, \cdot ;)$ denotes the discretization of the remaining terms, namely the convection terms and the zeroth-order terms. It is different from the standard DG discretization of convection terms, as it involves both upwind values of the solution and downwind value of the test functions.

$$
\begin{array}{r}
B_{c q}\left(c_{h}, w_{h} ; \mathbf{u}_{h}\right)=\frac{1}{2}\left(\left(\mathbf{u}_{h} \cdot \nabla c_{h}, w_{h}\right)_{\mathcal{E}_{h}}-\left(\mathbf{u}_{h} c_{h}, \nabla w_{h}\right)_{\mathcal{E}_{h}}+\left(\left(q^{I}+q^{P}\right) c_{h}, w_{h}\right)\right. \\
\left.+\left(c_{h}^{\mathrm{up}} \mathbf{u}_{h} \cdot \mathbf{n}_{e},\left[w_{h}\right]\right)_{\Gamma_{h}}-\left(w_{h}^{\mathrm{down}} \mathbf{u}_{h} \cdot \mathbf{n}_{e},\left[c_{h}\right]\right)_{\Gamma_{h}}\right)
\end{array}
$$

The upwind value (resp. downwind value) of a discontinuous function $w_{h}$ with respect to $\mathbf{u}_{h} \cdot \mathbf{n}_{e}$ is denoted by $w_{h}^{\text {up }}$ (resp. $\left.w_{h}^{\text {down }}\right)$.

$$
w_{h}^{\mathrm{up}}= \begin{cases}\left.w_{h}\right|_{E_{+}^{e}} & \text { if } \mathbf{u}_{h} \cdot \mathbf{n}_{e}>0 \\ \left.w_{h}\right|_{E_{-}^{e}} & \text { if } \mathbf{u}_{h} \cdot \mathbf{n}_{e} \leq 0\end{cases}
$$

We then set

$$
B\left(c_{h}, w_{h} ; \mathbf{u}_{h}\right)=B_{d}\left(c_{h}, w_{h} ; \mathbf{u}_{h}\right)+B_{c q}\left(c_{h}, w_{h} ; \mathbf{u}_{h}\right), \quad \forall c_{h}, w_{h}, \mathbf{u}_{h} .
$$

With this notation the continuous-in-time numerical scheme becomes: for all $t>0$ find $\mathbf{u}_{h}(t) \in \mathbf{U}_{h}, p_{h}(t) \in P_{h}, c_{h}(t) \in C_{h}$, satisfying

$$
\begin{aligned}
\left(\mathbf{K}^{-1}\left(c_{h}\right) \mathbf{u}_{h}, \mathbf{v}_{h}\right)-\left(p_{h}, \operatorname{div}\left(\mathbf{v}_{h}\right)\right) & =\left(\rho\left(c_{h}\right) \mathbf{g}, \mathbf{v}_{h}\right), \\
\left(q_{h}, \operatorname{div}\left(\mathbf{u}_{h}\right)\right) & =\left(q^{I}-q^{P}, q_{h}\right), \\
\left(\phi \partial_{t} c_{h}, w_{h}\right)+B\left(c_{h}, w_{h} ; \mathbf{u}_{h}\right) & =\left(\hat{c} q^{I}, w_{h}\right), \\
\left(c_{h}(0), w_{h}\right) & =\left(c_{0}, w_{h}\right),
\end{aligned}
$$

for all $\mathbf{v}_{h} \in \mathbf{U}_{h}, q_{h} \in P_{h}$, and $w_{h} \in C_{h}$.

\section{Fully-discrete scheme and decoupling algorithm}

Solving the flow and concentration equations with a fully implicit monolithic scheme can be computationally expensive. We choose to solve two sets of decoupled linear systems namely (9)-(10) and (11) sequentially. Let $\left\{t^{n}\right\}_{n=0}^{N}$ be a uniform partition of $[0, T]$, with time-step $\Delta t>0$. In the sequential updating, no time discretization is required for the discretization system (9)-(10) as it does not depend on time explicitly. For the time discretization of (11), we apply high order implicit Runge-Kutta methods to advance in time over each time interval $\left[t_{n-1}, t_{n}\right]$. 
However, difficulties occur if we want to maintain the high order in time convergence for all the unknowns. If the velocity is not very sensitive to time fluctuations we can simply use $\mathbf{u}_{h}\left(\cdot, t_{n-1}\right)$ which is nothing but a first-order approximation of the velocity in time, although this most likely causes deterioration of the high order convergence in time for the concentration. It is possible to use extrapolation to approximate $\mathbf{u}_{h}$ at the intermediate points using the previously computed $\mathbf{u}_{h}[4,14,9]$. In our work, we introduce an algorithm that is based on a class of implicit Runge-Kutta methods. These methods share the property that the upper triangular entries of the corresponding Butcher's tables are all zero. For each intermediate concentration $c_{h}^{(i)}$ we take advantage of the lower triangular structure of the Runge-Kutta method. We remark that an implicit Runge-Kutta method with a dense Butcher table is more computationally expensive. The algorithm is presented below.

\section{Algorithm 1.}

For all $n \leq N$ with $t_{N}=T$ and $\mathbf{v}_{h} \in \mathbf{U}_{h}, q_{h} \in P_{h}$, and $w_{h} \in C_{h}$.

$$
\text { Set } c_{h}^{(0)}=c_{h}\left(\cdot, t_{n-1}\right) \text {. }
$$

Let $i$ go from 1 to $s-1$ where $s-1$ is the number of intermediate points.

Find $\left(\mathbf{u}_{h}^{(i)}, p_{h}^{(i)}\right) \in\left(\mathbf{U}_{h}, P_{h}\right)$ such that

$$
\begin{array}{r}
\left(\mathbf{K}^{-1}\left(c_{h}^{(0)}\right) \mathbf{u}_{h}^{(i)}, \mathbf{v}_{h}\right)-\left(p_{h}^{(i)}, \operatorname{div}\left(\mathbf{v}_{h}\right)\right)=\left(\rho\left(c_{h}^{(0)}\right) \mathbf{g}, \mathbf{v}_{h}\right) \\
\left(q_{h}, \operatorname{div}\left(\mathbf{u}_{h}^{(i)}\right)\right)=\left(\left(q^{I}-q^{P}\right)\left(t_{n}^{(i)}\right), q_{h}\right)
\end{array}
$$

Find $c_{h}^{(i)} \in C_{h}$ by solving

$$
\left(\phi \frac{c_{h}^{(i)}-c_{h}^{n-1}}{\Delta t}, w_{h}\right)+\sum_{j=1}^{i} a_{i, j} B\left(c_{h}^{(j)}, w_{h} ; \mathbf{u}_{h}^{(j)}\right)=\sum_{j=1}^{i} a_{i, j}\left(\left(\hat{c} q^{I}\right)\left(t_{n}^{(j)}\right), w_{h}\right)
$$

Then, find $\left(\mathbf{u}_{h}^{(s)}, p_{h}^{(s)}\right) \in\left(\mathbf{U}_{h}, P_{h}\right)$ such that

$$
\begin{array}{r}
\left(\mathbf{K}^{-1}\left(c_{h}^{(0)}\right) \mathbf{u}_{h}^{(s)}, \mathbf{v}_{h}\right)-\left(p_{h}^{(s)}, \operatorname{div}\left(\mathbf{v}_{h}\right)\right)=\left(\rho\left(c_{h}^{(0)}\right) \mathbf{g}, \mathbf{v}_{h}\right) \\
\left(q_{h}, \operatorname{div}\left(\mathbf{u}_{h}^{(s)}\right)\right)=\left(\left(q^{I}-q^{P}\right)\left(t_{n}^{(s)}\right), q_{h}\right)
\end{array}
$$

Update $c_{h}\left(\cdot, t_{n}\right)=c_{h}^{n}$ by solving

$$
\left(\phi \frac{c_{h}^{n}-c_{h}^{n-1}}{\Delta t}, w_{h}\right)+\sum_{i=1}^{s-1} b_{i} B\left(c_{h}^{(i)}, w_{h} ; \mathbf{u}_{h}^{(i)}\right)=\sum_{i=1}^{s-1} b_{i}\left(\left(\hat{c} q^{I}\right)\left(t_{n}^{(i)}\right), w_{h}\right)
$$

and set $n=n+1$. 
The intermediate time steps are defined as $t_{n}^{(i)}=t_{n-1}+d_{i} \Delta t$. The coefficients $a_{i, j}$ 's, $b_{i}$ 's and $d_{i}$ 's are taken from the Butcher's table for the Runge-Kutta method:

$$
\begin{array}{l|l}
d & \mathbf{A} \\
\hline & b^{\top}
\end{array}
$$

We will use in our numerical results the implicit Runge-Kutta methods with Butcher tables given in Table 1 and Table 2.

\begin{tabular}{r|rr|r}
1 & 1
\end{tabular}$\quad$\begin{tabular}{r|r}
$1 / 2$ & $1 / 2$ \\
\hline & 1
\end{tabular}$\quad \begin{aligned} & 1 \\
& \end{aligned}$

Table 1: First-order implicit Euler (left) and second-order Gauss I method (right)

$$
\begin{array}{r|rrr|rrr}
1 / 3 & 1 / 3 & 0 & 0 & 0 & 0 & 0 \\
1 & 1 & 0 & 1 / 2 & 1 / 4 & 1 / 4 & 0 \\
& 3 / 4 & 1 / 4 & 1 & 0 & 1 & 0 \\
\hline & & & 1 / 6 & 2 / 3 & 1 / 6
\end{array}
$$

Table 2: Third-order Radau II method (left) and fourth-order Lobatto III method (right)

The theoretical convergence of our method is shown in [10]. For completness we recall the results below. We first rewrite the time-stepping scheme as a discontinuous Galerkin in time method. The following scheme is equivalent to the fully discrete scheme defined in Algorithm 1: find $\mathbf{u}_{h} \in \mathcal{P}_{0}\left[t^{n-1}, t^{n} ; \mathbf{U}_{h}\right]$, $p_{h} \in \mathcal{P}_{0}\left[t^{n-1}, t^{n} ; P_{h}\right], c_{h} \in P_{\ell}\left[t^{n-1}, t^{n} ; C_{h}\right]$, satisfying

$$
\begin{gathered}
\int_{t_{n-1}}^{t_{n}}\left(\left(\mathbf{K}^{-1}\left(c_{h}\right) \mathbf{u}_{h}, \mathbf{v}_{h}\right)-\left(p_{h}, \operatorname{div}\left(\mathbf{v}_{h}\right)\right)\right)=\int_{t_{n-1}}^{t_{n}}\left(\rho\left(c_{h}\right) \mathbf{g}, \mathbf{v}_{h}\right) \\
\int_{t_{n-1}}^{t_{n}}\left(q_{h}, \operatorname{div}\left(\mathbf{u}_{h}\right)\right)=\int_{t_{n-1}}^{t_{n}}\left(q^{I}-q^{P}, q_{h}\right) \\
\int_{t_{n-1}}^{t_{n}}\left(\left(\phi \partial_{t} c_{h}, w_{h}\right)+B\left(c_{h}, w_{h} ; \mathbf{u}_{h}\right)\right)+\left(\left[c_{h}^{n-1}\right]_{t}, \phi w_{h+}^{n-1}\right)=\int_{t_{n-1}}^{t_{n}}\left(\hat{c} q^{I}, w_{h}\right),
\end{gathered}
$$

The concentrations are discontinuous polynomials of degree $\ell$ in time, which yields a Runge-Kutta method of order $\ell+1$. We have also introduced the notation for the jump of a solution in time:

$$
v_{+}^{n}=\lim _{\epsilon \downarrow 0} v\left(\cdot, t_{n}+\epsilon\right), v_{-}^{n}=\lim _{\epsilon \downarrow 0} v\left(\cdot, t_{n}-\epsilon\right),\left[v^{n}\right]_{t}=v_{+}^{n}-v_{-}^{n} .
$$


We also define a weak solution to the miscible displacement problem [10]: $(\mathbf{u}, p, c) \in L^{\infty}\left[0, T ; H_{0}(\Omega, \operatorname{div})\right] \times L^{\infty}\left[0, T ; L_{0}^{2}(\Omega)\right] \times L^{2}\left[0, T ; H^{1}(\Omega)\right]$ and

$$
\begin{gathered}
\int_{0}^{T}\left(\mathbf{K}^{-1}(c) \mathbf{u}, \mathbf{v}\right)-(p, \operatorname{div}(\mathbf{v}))=\int_{0}^{T}(\rho(c) \mathbf{g}, \mathbf{v}) \\
\int_{0}^{T}(q, \operatorname{div}(\mathbf{u}))=\int_{0}^{T}\left(q^{I}-q^{P}, q\right) \\
\int_{0}^{T}\left(-\left(\phi c, \partial_{t} w\right)+(\mathbf{D}(\mathbf{u}) \nabla c-(1 / 2) c \mathbf{u}, \nabla w)\right. \\
\left.+(1 / 2)(\mathbf{u} \cdot \nabla c, w)+(1 / 2)\left(\left(q^{I}+q^{P}\right) c, w\right)\right)=\left(\phi c_{0}, w(0)\right)+\int_{0}^{T}\left(q^{I} \hat{c}, w\right),
\end{gathered}
$$

for all $(\mathbf{v}, q) \in L^{1}[0, T ; H(\Omega, \operatorname{div})] \times L^{1}\left[0, T ; L_{0}^{2}(\Omega)\right]$ and for all

$$
w \in\left\{w \in L^{4}\left[0, T ; W^{1,4}(\Omega)\right] \cap H^{1}\left[0, T ; H^{1}(\Omega)^{\prime}\right]: w(T)=0\right\} .
$$

We briefly state the convergence theorem below.

Theorem 1. Let $\left\{\mathcal{E}_{h}\right\}_{h>0}$ be a regular family of meshes and let $\left\{\mathbf{u}_{h}, p_{h}, c_{h}\right\}_{h>0}$ be solutions of the discrete scheme (13)-(15). If the time step $\Delta t$ tends to zero with the mesh parameter $h$, then, upon passage to a subsequence, $\left\{\mathbf{u}_{h}, p_{h}, c_{h}\right\}_{h>0}$ converges strongly in $L^{2}[0, T ; H(\Omega ; \operatorname{div})] \times L^{2}\left[0, T ; L^{2}(\Omega)\right] \times$ $L^{2}\left[0, T ; L^{2}(\Omega)\right]$. In particular, the numerical solutions of the SIPG discretization converge to a solution of the weak statement (16)-(18).

\section{Numerical results}

In this section we present both numerical results for analytical and physical solutions of the miscible displacement problem. We will compare the solutions obtained by varying the order of the method in time and in space. We begin with some notation. Let $\mathrm{RT}_{k}-\mathrm{SIPG}_{r}$ denote the method that combines the mixed finite element method with the Raviart-Thomas space $\mathrm{RT}_{k}$ and the symmetric interior penalty method $(\epsilon=-1)$ with the space of discontinuous polynomials of degree $r$ (see (4)-(6)). Similarly, we define the method $\mathrm{RT}_{k}-\mathrm{NIPG}_{r}$ and the method $\mathrm{RT}_{k}-\mathrm{IIPG}_{r}$ for the choice $\epsilon=1$ and $\epsilon=0$ in (7) respectively. The implementation of the method has been carried out 
in the DUNE framework [1]. Finally we remark that the scheme described in [9] uses a first order backward Euler method in time and discontinuous Galerkin in space for both pressure-velocity and concentration equations. In addition the penalty term is independent of the velocity, the convection term is treated with a standard upwind technique, and slope limiters are needed for the method described in [9].

\subsection{Analytical Problem and Convergence Study}

Consider the miscible displacement problem in $\Omega=(0,1)^{2}$ with the following analytical solutions:

$$
\begin{aligned}
& p(x, y, t)=\left(2-e^{-x}\left(1+x+x^{2}\right)-e^{-y}\left(1+y+y^{2}\right)\right) e^{\frac{\pi t}{2}}, \\
& c(x, y, t)=\frac{1}{2}\left(\sin (2 \pi x)^{2}+\cos (2 \pi y)^{2}\right) \sin \left(\frac{\pi t}{2}\right) .
\end{aligned}
$$

For the diffusion-dispersion tensor we use the semi-empirical relation:

$$
\mathbf{D}(\mathbf{u})=d_{m} \mathbf{I}+|\mathbf{u}|\left(\alpha_{l} E(\mathbf{u})+\alpha_{t}(\mathbf{I}-E(\mathbf{u}))\right),
$$

where $E(\mathbf{u})=\frac{\mathbf{u} \mathbf{u}^{T}}{|\mathbf{u}|^{2}}$ and we set,

$$
d_{m}=1.8 \times 10^{-7}, \alpha_{l}=1,8 \times 10^{-5} \text { and } \alpha_{t}=1.8 \times 10^{-6} .
$$

The parameters $d_{m}, \alpha_{l}$ and $\alpha_{t}$ are the molecular diffusion, longitudinal dispersivity and transverse dispersivity respectively. The other parameters are

$$
\phi=0.2, \mathbf{K}(c)=\frac{9.44 \times 10^{-3}}{\left(c(2.9)^{-0.25}+(1-c)(5.8)^{-0.25}\right)^{-4}}, \mathbf{g}=0 .
$$

We fix the time-discretization method to be the fourth order Lobatto III method. The time-step is equal to 0.01. For the discretization in space we use $\mathrm{RT}_{0}-\mathrm{NIPG}_{1}, \mathrm{RT}_{1}-\mathrm{NIPG}_{2}$, and $\mathrm{RT}_{2}-\mathrm{NIPG}_{3}$, which are first, second and third order respectively. We compute the errors between the exact solutions and the numerical solutions at the final time $T=0.5$ and for a sequence of uniformly refined meshes.

$$
\begin{gathered}
L^{2} \text { error for pressure : }\left\|p(T)-p_{h}^{N}\right\|_{L^{2}(\Omega)} \\
L^{2} \text { error for velocity : }\left\|\mathbf{u}(T)-\mathbf{u}_{h}^{N}\right\|_{L^{2}(\Omega)} \\
L^{2} \text { error for concentration : }\left\|c(T)-c_{h}^{N}\right\|_{L^{2}(\Omega)} \\
\text { Energy error for concentration : }\left\|\nabla\left(c(T)-c_{h}^{N}\right)\right\|_{L^{2}\left(\mathcal{E}_{h}\right)}
\end{gathered}
$$


Table 3 gives the errors and convergence rates for the method $\mathrm{RT}_{0}-\mathrm{NIPG}_{1}$. Similarly, Table 4 and Table 5 show the errors and convergence rates for the method $\mathrm{RT}_{1}-\mathrm{NIPG}_{2}$ and $\mathrm{RT}_{2}-\mathrm{NIPG}_{3}$ respectively. We observe that for a given mesh size $h$, the errors decrease as the order of the method increases. We also observe the expected optimal rates in space:

$$
\begin{gathered}
\left\|p(T)-p_{h}^{N}\right\|_{L^{2}(\Omega)}=\mathcal{O}\left(h^{k+1}\right) \\
\left\|\mathbf{u}(T)-\mathbf{u}_{h}^{N}\right\|_{L^{2}(\Omega)}=\mathcal{O}\left(h^{k+1}\right) \\
\left\|c(T)-c_{h}^{N}\right\|_{L^{2}(\Omega)}=\mathcal{O}\left(h^{r+1}\right) \\
\left\|\nabla\left(c(T)-c_{h}^{N}\right)\right\|_{L_{2}\left(\mathcal{E}_{h}\right)}=\mathcal{O}\left(h^{r}\right)
\end{gathered}
$$

The deterioration of the rates in the last two lines in Table 5 is due to the fact that the time-step is kept constant throughout the simulations. The time-discretization error dominates when the spatial errors are too small. If we decrease the time step, we recover the optimal rates. In the next two tables we show that the choice of the symmetrization parameter $\epsilon$ in (7) does not have a visible effect on the errors and rates. We repeat the experiments above with either the SIPG method $(\epsilon=-1)$ or the IIPG method $(\epsilon=0)$. Errors and rates are computed for the last two finer meshes (see Table 6 and Table 7).

Next, we compute the convergence rates with respect to time by fixing the mesh $h=1 / 64$ and by varying the time-step $\Delta t$ and the order of the RungeKutta method. The spatial discretization is the method $\mathrm{RT}_{2}-\mathrm{NIPG}_{3}$. The errors in the concentration are computed at the final time $T=1$. Table 8 shows the numerical errors and rates obtained as the time-step decreases. The results confirm the first-order convergence rate of the implicit Euler method. Similarly Table 9 and Table 10 show the errors and rates for the time-stepping method of second and third order respectively. We observe that our decoupling strategy preserves the high order in time method. We also note that for a fixed time-step, the accuracy of the numerical solution increases with the order of the method. Finally we observe a drop in the convergence rate for Table 10 while going from $\Delta t=2^{-4}$ to $\Delta t=2^{-5}$ due to the spatial discretization error. If the mesh is finer than $h=1 / 64$, optimal convergence rates are recovered. 


\subsection{Physical Problem}

\subsubsection{Homogeneous permeability}

Now, we turn our attention to a physical problem over the spatial domain $(0,1)^{2}$. We fix the injection concentration to be $\hat{c}=1$ and initial concentration $c_{0}=0$. For the injection source and production sink we choose

$$
\int_{\Omega} q^{I}=\int_{\Omega} q^{P}=0.018
$$

where $q^{I}$ is piecewise constant on $[0,0.1] \times[0,0.1]$ and $q^{I}=0$ elsewhere and $q^{P}$ is piecewise constant on $[0.9,1] \times[0.9,1]$ and $q^{P}=0$ elsewhere. The physical parameters are

$$
\phi=0.2, \mathbf{K}(c)=\frac{9.44 \times 10^{-3}}{1+(0.0524 c)^{4.75}}, \mathbf{g}=0 .
$$

The other parameters are the same as in (19) and (20).

We apply our algorithm to simulate the miscible displacement and plot the fluid profile from $t=0$ to $t=10$ as follows with $\mathrm{RT}_{1}-\mathrm{NIPG}_{1}$ and $\mathrm{RT}_{2}$ $\mathrm{NIPG}_{2}$. The mesh is made of 1024 square elements and the time step is $\Delta t=0.05$. We also vary the order of the method in time.

In Figure 1, snapshots of the fluid concentration are given with the first order method in time, and with second and third order in space. One can observe the increase of the quality of the simulations and the sharpness of the concentration fronts as we use higher order approximations in space.

We repeat the experiments and use the second, third and fourth order method in time in Figure 2, Figure 3 and Figure 4 respectively. We observe that the location of the concentration fronts is the same as we vary the order of the method. This is evidence of the convergence of the method. We also note that the fronts are sharper as the order of the method increases.

To better see this, we show in Fig. 5 the concentration profile, at time $t=$ 7.5 , plotted along the diagonal $y=x$ for different orders in time. The spatial discretization is fixed, and is the $\mathrm{RT}_{2}-\mathrm{NIPG}_{2}$ method. The plots illustrate the effect of using higher order approximations in time. On one hand, we observe the localized overshoot and undershoot phenomena due to the high order approximation. On the other hand, we have gained considerable accuracy globally using the high order approximations in time and the overshoot and undershoot phenomena remain stable and small throughout the simulation. 


\subsubsection{Homogeneous permeability with a discontinuous lens}

In this example, the porous medium has a square inclusion with a lower permeability. In Figure 6, we set the permeability of the shaded area to be 1000 times smaller than in the rest of the domain. The remaining parameters are the same as in the previous example. We simulate the miscible displacement problem using spatial discretization $\mathrm{RT}_{2}-\mathrm{NIPG}_{2}$ with 4096 elements. For the time discretization, we use Implicit Euler and Gauss I methods in time with $\Delta t=0.05$ and the final simulation time is $T=10$. We compare the numerical concentrations at different times in Figure 7. We observe that both methods capture well the region of discontinuity of the permeability. The higher order in time method exhibits less diffusion in the concentration fronts.

\subsubsection{Heterogeneous permeabilities}

In the last two examples, we consider the permeability fields from the SPE 10 benchmark problem [17]. The first permeability field is from a Tarbert formation while the second is from an Upper Ness formation. Fig. 8 shows the permeability values in a logarithmic scale. The Upper Ness formation is a more challenging field as there are intricate fractures in the medium and the range of the permeability values is larger than for the Tarbert case. We apply the second order in space $\mathrm{RT}_{2}-\mathrm{NIPG}_{2}$ with the second order Gauss I method in time. Snapshots of the concentration at different times for the Tarbet case are shown in Figure 9 whereas the solutions for the Upper Ness formation are shown in Figure 10. We observe in both cases, that the solvent fluid reaches the production well by sweeping the regions with highest permeability values. As expected, the boundary of these regions is less smooth for the porous medium of Upper Ness type. Our method captures the very small regions of lower permeability and the concentration fronts remain sharp.

\section{Conclusion}

In this work we propose a method of high order for solving the miscible displacement problem. Raviart-Thomas elements are used for approximating the pressure and velocity, and discontinuous piecewise polynomials are used for the concentration. Our numerical results show the convergence and robustness of the numerical solution on homogeneous and heterogeneous media. 


\section{References}

[1] P. Bastian, M. Blatt, A. Dedner, C. Engwer, R. Klöfkorn, M. Ohlberger, O. Sander, A Generic Grid Interface for Parallel and Adaptive Scientific Computing. Part I: Abstract Framework, . Computing 82(2-3), (2008) 103-119.

[2] R. Ewing, T. Russell, Efficient time-stepping methods for miscible displacement problems in porous media, SIAM J. Numer. Anal. 19 (1982) $1-67$.

[3] R. Ewing, M. Wheeler, Galerkin methods for miscible displacement problems in porous media, SIAM J. Numer. Anal. 17 (1980) 351-365.

[4] T. Russell, Time stepping along characteristics with incomplete iteration for a Galerkin approximation of miscible displacement in porous media, SIAM J. Numer. Anal. 22 (5) (1985) 970-1013.

[5] J. Douglas, R. E. Ewing, M. Wheeler, A time-discretization procedure for a mixed finite element approximation of miscible displacement in porous media, R.A.I.R.O. Numerical Analysis 17 (3) (1983) 249-265.

[6] Y. Epshteyn, B. B. Rivière, Convergence of high order methods for miscible displacement, International Journal of Numerical Analysis and Modeling 5 (2008) 47-63.

[7] M. Ohlberger, Convergence of a mixed finite element - finite volume method for the two phase flow in porous media, East-Weat J. of Numerical Mathematics 5 (1997) 183-210.

[8] S. Sun, B. Rivière, M. Wheeler, A combined mixed finite element and discontinuous Galerkin method for miscible displacement problem in porous media, Recent Progress in Computational and Applied PDEs (2002) 323-348.

[9] B. Rivière, M. Wheeler, Discontinuous Galerkin methods for flow and transport problems in porous media, Communications in Numerical Methods in Engineering 18 (2002) 63-68.

[10] J. Li, B. Riviere, N. Walkington, Convergence of a high order method in time and space for the miscible displacement equations, ESAIM: Mathematical Modelling and Numerical AnalysisSubmitted. 
[11] S. Gottlieb, G. Wei, S. Zhao, A unified discontinuous Galerkin framework for time integration, preprint.

[12] S. Zhao, G. W. Wei, A unified discontinuous Galerkin framework for time integration, Mathematical Methods in the Applied Sciences, DOI: 10.1002/mma.2863.

[13] S. Bartels, M. Jensen, R. Müller, Discontinuous Galerkin finite element convergence for incompressible miscible displacement problems of low regularity, SIAM J. Numer. Anal. 47 (2009) 3720-3743.

[14] M. Jensen, R. Müller, Stable Crank-Nicolson discretisation for incompressible miscible displacement problems of low regularity, Numerical Mathematics and Advanced Applications 2009 (2010) 469-477.

[15] B. Riviere, N. Walkington, Convergence of a discontinuous Galerkin method for the miscible displacement under low regularity, SIAM J. Numer. Anal. 49 (2011) 1085-1110.

[16] F. Brezzi, J. Douglas, M. Fortin, L. Marini, Efficient rectangular mixed finite elements in two and three space variables, RAIRO Model. Math. Anal. Numer. 21 (1987) 581-604.

[17] M. Christie, M. Blunt, Tenth SPE comparative solution project: a comparison of upscaling techniques, SPE Reservoir Engineering and Evaluation 4 (2001) 308-317. 


\begin{tabular}{|c|c|c|c|c|}
\hline \multicolumn{5}{|c|}{ Pressure and velocity } \\
\hline$h$ & $\left\|p-p_{h}\right\|_{L^{2}(\Omega)}$ & Cvg. rate & $\left\|\mathbf{u}-\mathbf{u}_{h}\right\|_{L^{2}(\Omega)}$ & Cvg. rate \\
\hline $2^{-3}$ & $1.89 \mathrm{e}-2$ & 1.47 & $3.65 \mathrm{e}-5$ & 1.44 \\
\hline $2^{-4}$ & $7.44 \mathrm{e}-3$ & 1.35 & $1.60 \mathrm{e}-5$ & 1.19 \\
\hline $2^{-5}$ & $3.36 \mathrm{e}-3$ & 1.15 & $7.70 \mathrm{e}-6$ & 1.06 \\
\hline $2^{-6}$ & $1.62 \mathrm{e}-3$ & 1.05 & $3.81 \mathrm{e}-6$ & 1.02 \\
\hline $2^{-7}$ & $8.05 \mathrm{e}-4$ & 1.01 & $1.89 \mathrm{e}-6$ & 1.00 \\
\hline \hline \multicolumn{5}{|c|}{ Concentration } \\
\hline$h$ & $\left\|c-c_{h}\right\|_{L^{2}(\Omega)}$ & Cvg. rate & $\left\|\nabla c-\nabla c_{h}\right\|_{L^{2}\left(\mathcal{E}_{h}\right)}$ & Cvg. rate \\
\hline $2^{-3}$ & $1.57 \mathrm{e}-2$ & 0.44 & $9.78 \mathrm{e}-1$ & -1.01 \\
\hline $2^{-4}$ & $4.03 \mathrm{e}-3$ & 1.96 & $5.00 \mathrm{e}-1$ & 0.96 \\
\hline $2^{-5}$ & $1.02 \mathrm{e}-3$ & 1.99 & $2.52 \mathrm{e}-1$ & 0.99 \\
\hline $2^{-6}$ & $2.57 \mathrm{e}-4$ & 1.99 & $1.26 \mathrm{e}-1$ & 1.00 \\
\hline $2^{-7}$ & $6.59 \mathrm{e}-5$ & 1.96 & $6.34 \mathrm{e}-2$ & 0.99 \\
\hline
\end{tabular}

Table 3: Errors and rates for the method with $\mathrm{RT}_{0}-\mathrm{NIPG} \mathrm{G}_{1}$ and Lobatto III methods.

\begin{tabular}{|c|c|c|c|c|}
\hline \multicolumn{5}{|c|}{ Pressure and velocity } \\
\hline$h$ & $\left\|p-p_{h}\right\|_{L^{2}(\Omega)}$ & Cvg. rate & $\left\|\mathbf{u}-\mathbf{u}_{h}\right\|_{L^{2}(\Omega)}$ & Cvg. rate \\
\hline $2^{-3}$ & $4.74 \mathrm{e}-3$ & 1.71 & $6.19 \mathrm{e}-6$ & 1.16 \\
\hline $2^{-4}$ & $1.30 \mathrm{e}-3$ & 1.86 & $1.55 \mathrm{e}-6$ & 2.00 \\
\hline $2^{-5}$ & $3.44 \mathrm{e}-4$ & 1.93 & $3.86 \mathrm{e}-7$ & 2.00 \\
\hline $2^{-6}$ & $8.83 \mathrm{e}-5$ & 1.96 & $9.65 \mathrm{e}-8$ & 2.00 \\
\hline $2^{-7}$ & $2.77 \mathrm{e}-5$ & 1.96 & $2.41 \mathrm{e}-8$ & 2.00 \\
\hline \hline \multicolumn{5}{|c|}{ Concentration } \\
\hline$h$ & $\left\|c-c_{h}\right\|_{L^{2}(\Omega)}$ & Cvg. rate & $\left\|\nabla c-\nabla c_{h}\right\|_{L^{2}\left(\mathcal{E}_{h}\right)}$ & Cvg. rate \\
\hline \hline $2^{-3}$ & $2.10 \mathrm{e}-3$ & 3.34 & $2.17 \mathrm{e}-1$ & 2.35 \\
\hline $2^{-4}$ & $2.68 \mathrm{e}-4$ & 2.97 & $5.53 \mathrm{e}-2$ & 1.97 \\
\hline $2^{-5}$ & $3.38 \mathrm{e}-5$ & 2.99 & $1.39 \mathrm{e}-2$ & 2.00 \\
\hline $2^{-6}$ & $4.27 \mathrm{e}-6$ & 2.98 & $3.46 \mathrm{e}-3$ & 2.00 \\
\hline $2^{-7}$ & $7.13 \mathrm{e}-7$ & 2.58 & $8.67 \mathrm{e}-4$ & 2.00 \\
\hline
\end{tabular}

Table 4: Errors and rates for the method combining $\mathrm{RT}_{1}-\mathrm{NIPG}_{2}$ and Lobatto III. 


\begin{tabular}{|c|c|c|c|c|}
\hline \multicolumn{5}{|c|}{ Pressure and velocity } \\
\hline$h$ & $\left\|p-p_{h}\right\|_{L^{2}(\Omega)}$ & Cvg. rate & $\left\|\mathbf{u}-\mathbf{u}_{h}\right\|_{L^{2}(\Omega)}$ & Cvg. rate \\
\hline $2^{-3}$ & $2.56 \mathrm{e}-4$ & 2.87 & $8.47 \mathrm{e}-7$ & 3.34 \\
\hline $2^{-4}$ & $3.44 \mathrm{e}-5$ & 2.89 & $1.04 \mathrm{e}-7$ & 3.03 \\
\hline $2^{-5}$ & $4.93 \mathrm{e}-6$ & 2.80 & $1.30 \mathrm{e}-8$ & 3.00 \\
\hline $2^{-6}$ & $1.15 \mathrm{e}-6$ & 2.10 & $1.63 \mathrm{e}-9$ & 2.99 \\
\hline $2^{-7}$ & $6.89 \mathrm{e}-7$ & 0.73 & $2.85 \mathrm{e}-10$ & 2.51 \\
\hline \hline \multicolumn{5}{|c|}{ Concentration } \\
\hline$h$ & $\left\|c-c_{h}\right\|_{L^{2}(\Omega)}$ & Cvg. rate & $\left\|\nabla c-\nabla c_{h}\right\|_{L^{2}\left(\mathcal{E}_{h}\right)}$ & Cvg. rate \\
\hline $2^{-3}$ & $2.08 \mathrm{e}-4$ & 1.73 & $3.15 \mathrm{e}-2$ & 0.51 \\
\hline $2^{-4}$ & $1.33 \mathrm{e}-5$ & 3.97 & $4.02 \mathrm{e}-3$ & 2.97 \\
\hline $2^{-5}$ & $9.68 \mathrm{e}-7$ & 3.78 & $5.07 \mathrm{e}-4$ & 2.99 \\
\hline $2^{-6}$ & $4.76 \mathrm{e}-7$ & 1.02 & $6.41 \mathrm{e}-5$ & 2.98 \\
\hline $2^{-7}$ & $4.73 \mathrm{e}-7$ & 0.01 & $1.15 \mathrm{e}-5$ & 2.48 \\
\hline
\end{tabular}

Table 5: Errors and rates for the method with $\mathrm{RT}_{2}-\mathrm{NIPG}_{3}$ and Lobatto III methods.

\begin{tabular}{|c|c|c|c|c|c|}
\hline & $h$ & $\left\|p-p_{h}\right\|_{L^{2}(\Omega)}$ & rate & $\left\|\mathbf{u}-\mathbf{u}_{h}\right\|_{L^{2}(\Omega)}$ & rate \\
\hline \multirow{2}{*}{$\mathrm{RT}_{0}-\mathrm{SIPG}_{1}$} & $2^{-4}$ & $7.44 \mathrm{e}-3$ & - & $1.60 \mathrm{e}-5$ & - \\
\cline { 2 - 6 } & $2^{-5}$ & $3.36 \mathrm{e}-3$ & 1.15 & $7.70 \mathrm{e}-6$ & 1.06 \\
\hline \multirow{2}{*}{$\mathrm{RT}_{0}-\mathrm{IIPG}_{1}$} & $2^{-4}$ & $7.44 \mathrm{e}-3$ & - & $1.60 \mathrm{e}-5$ & - \\
\cline { 2 - 6 } & $2^{-5}$ & $3.36 \mathrm{e}-3$ & 1.15 & $7.70 \mathrm{e}-6$ & 1.06 \\
\hline \multirow{2}{*}{$\mathrm{RT}_{1}-\mathrm{SIPG}_{2}$} & $2^{-4}$ & $1.30 \mathrm{e}-3$ & - & $1.55 \mathrm{e}-6$ & - \\
\cline { 2 - 6 } & $2^{-5}$ & $3.44 \mathrm{e}-4$ & 1.93 & $3.86 \mathrm{e}-7$ & 2.00 \\
\hline \multirow{2}{*}{$\mathrm{RT}_{1}-\mathrm{IIPG}_{2}$} & $2^{-4}$ & $1.30 \mathrm{e}-3$ & - & $1.55 \mathrm{e}-6$ & - \\
\cline { 2 - 6 } & $2^{-5}$ & $3.44 \mathrm{e}-4$ & 1.93 & $3.86 \mathrm{e}-7$ & 2.00 \\
\hline \multirow{2}{*}{$\mathrm{RT}_{2}-\mathrm{SIPG}_{3}$} & $2^{-4}$ & $3.44 \mathrm{e}-5$ & - & $1.04 \mathrm{e}-7$ & - \\
\cline { 2 - 6 } & $2^{-5}$ & $4.93 \mathrm{e}-6$ & 2.80 & $1.30 \mathrm{e}-8$ & 3.00 \\
\hline \multirow{2}{*}{$\mathrm{RT}_{2}-\mathrm{IIPG}_{3}$} & $2^{-4}$ & $3.44 \mathrm{e}-5$ & - & $1.04 \mathrm{e}-7$ & - \\
\cline { 2 - 6 } & $2^{-5}$ & $4.93 \mathrm{e}-6$ & 2.80 & $1.30 \mathrm{e}-8$ & 3.00 \\
\hline
\end{tabular}

Table 6: Pressure and velocity: errors and rates for $\mathrm{RT}_{k}-\mathrm{SIPG}_{r}$ and $\mathrm{RT}_{k}$-IIPG $\mathrm{G}_{r}$ methods and Lobatto III methods. 


\begin{tabular}{|c|c|c|c|c|c|}
\hline & $h$ & $\left\|c-c_{h}\right\|_{L^{2}(\Omega)}$ & rate & $\left\|\nabla c-\nabla c_{h}\right\|_{L^{2}\left(\mathcal{E}_{h}\right)}$ & rate \\
\hline \multirow{2}{*}{$\mathrm{RT}_{0}-\mathrm{SIPG}_{1}$} & $2^{-4}$ & $4.03 \mathrm{e}-3$ & - & $5.00 \mathrm{e}-1$ & - \\
\cline { 2 - 6 } & $2^{-5}$ & $1.02 \mathrm{e}-3$ & 1.99 & $2.52 \mathrm{e}-1$ & 0.99 \\
\hline \multirow{2}{*}{$\mathrm{RT}_{0}-\mathrm{IIPG}_{1}$} & $2^{-4}$ & $4.03 \mathrm{e}-3$ & - & $5.00 \mathrm{e}-1$ & - \\
\cline { 2 - 6 } & $2^{-5}$ & $1.02 \mathrm{e}-3$ & 1.99 & $2.52 \mathrm{e}-1$ & 0.99 \\
\hline \multirow{2}{*}{$\mathrm{RT}_{1}-\mathrm{SIPG}_{2}$} & $2^{-4}$ & $2.68 \mathrm{e}-4$ & - & $5.53 \mathrm{e}-2$ & - \\
\cline { 2 - 6 } & $2^{-5}$ & $3.38 \mathrm{e}-5$ & 2.99 & $1.39 \mathrm{e}-2$ & 2.00 \\
\hline \multirow{2}{*}{$\mathrm{RT}_{1}-\mathrm{IIPG}_{2}$} & $2^{-4}$ & $2.68 \mathrm{e}-4$ & - & $5.53 \mathrm{e}-2$ & - \\
\cline { 2 - 6 } & $2^{-5}$ & $3.38 \mathrm{e}-5$ & 2.99 & $1.39 \mathrm{e}-2$ & 2.00 \\
\hline \multirow{2}{*}{$\mathrm{RT}_{2}-\mathrm{SIPG}_{3}$} & $2^{-4}$ & $1.33 \mathrm{e}-5$ & - & $4.02 \mathrm{e}-3$ & - \\
\cline { 2 - 6 } & $2^{-5}$ & $9.68 \mathrm{e}-7$ & 3.78 & $5.07 \mathrm{e}-4$ & 2.99 \\
\hline \multirow{2}{*}{$\mathrm{RT}_{2}-\mathrm{IIPG}_{3}$} & $2^{-4}$ & $1.33 \mathrm{e}-5$ & - & $4.02 \mathrm{e}-3$ & - \\
\cline { 2 - 6 } & $2^{-5}$ & $9.68 \mathrm{e}-7$ & 3.78 & $5.07 \mathrm{e}-4$ & 2.99 \\
\hline
\end{tabular}

Table 7: Concentration: errors and rates for $\mathrm{RT}_{k}-\mathrm{SIPG}_{r}$ and $\mathrm{RT}_{k}-\mathrm{IIPG}_{r}$ methods and Lobatto III methods.

\begin{tabular}{|c|c|c|c|c|}
\hline \multicolumn{5}{|c|}{ Pressure and velocity } \\
\hline$\Delta t$ & $\left\|p-p_{h}\right\|_{L^{2}(\Omega)}$ & Cvg. rate & $\left\|\mathbf{u}-\mathbf{u}_{h}\right\|_{L^{2}(\Omega)}$ & Cvg. rate \\
\hline $2^{-1}$ & $3.66 \mathrm{e}-2$ & - & $3.32 \mathrm{e}-5$ & - \\
\hline $2^{-2}$ & $2.08 \mathrm{e}-2$ & 0.90 & $1.90 \mathrm{e}-5$ & 0.90 \\
\hline $2^{-3}$ & $1.11 \mathrm{e}-2$ & 0.95 & $1.02 \mathrm{e}-5$ & 0.95 \\
\hline $2^{-4}$ & $5.75 \mathrm{e}-3$ & 0.97 & $5.30 \mathrm{e}-6$ & 0.97 \\
\hline $2^{-5}$ & $2.93 \mathrm{e}-3$ & 0.99 & $2.70 \mathrm{e}-6$ & 0.99 \\
\hline \hline \multicolumn{5}{|c|}{ Concentration } \\
\hline$\Delta t$ & $\left\|c-c_{h}\right\|_{L^{2}(\Omega)}$ & Cvg. rate & $\left.\left\|\nabla c-\nabla c_{h}\right\|_{L^{2}\left(\mathcal{E}_{h}\right)}\right)$ & Cvg. rate \\
\hline $2^{-1}$ & $9.32 \mathrm{e}-2$ & - & $5.24 \mathrm{e}-1$ & - \\
\hline $2^{-2}$ & $5.37 \mathrm{e}-2$ & 0.89 & $3.02 \mathrm{e}-1$ & 0.89 \\
\hline $2^{-3}$ & $2.90 \mathrm{e}-2$ & 0.94 & $1.63 \mathrm{e}-1$ & 0.94 \\
\hline $2^{-4}$ & $1.51 \mathrm{e}-2$ & 0.97 & $8.48 \mathrm{e}-2$ & 0.97 \\
\hline $2^{-5}$ & $7.70 \mathrm{e}-3$ & 0.98 & $4.33 \mathrm{e}-2$ & 0.98 \\
\hline
\end{tabular}

Table 8: Errors and rates with implicit Euler time-stepping method and $\mathrm{RT}_{2}-\mathrm{NIPG}_{3}$. 


\begin{tabular}{|c|c|c|c|c|}
\hline \multicolumn{5}{|c|}{ Pressure and velocity } \\
\hline$\Delta t$ & $\left\|p-p_{h}\right\|_{L^{2}(\Omega)}$ & Cvg. rate & $\left\|\mathbf{u}-\mathbf{u}_{h}\right\|_{L^{2}(\Omega)}$ & Cvg. rate \\
\hline $2^{-1}$ & $6.44 \mathrm{e}-2$ & - & $6.21 \mathrm{e}-5$ & - \\
\hline $2^{-2}$ & $1.61 \mathrm{e}-2$ & 2.05 & $1.50 \mathrm{e}-5$ & 2.06 \\
\hline $2^{-3}$ & $3.87 \mathrm{e}-3$ & 2.01 & $3.59 \mathrm{e}-6$ & 2.01 \\
\hline $2^{-4}$ & $9.60 \mathrm{e}-4$ & 2.01 & $8.91 \mathrm{e}-7$ & 2.00 \\
\hline $2^{-5}$ & $2.38 \mathrm{e}-4$ & 2.05 & $2.22 \mathrm{e}-7$ & 1.99 \\
\hline \hline \multicolumn{5}{|c|}{ Concentration } \\
\hline$\Delta t$ & $\left\|c-c_{h}\right\|_{L^{2}(\Omega)}$ & Cvg. rate & $\left\|\nabla c-\nabla c_{h}\right\|_{L^{2}}\left(\mathcal{E}_{h}\right)$ & Cvg. rate \\
\hline $2^{-1}$ & $1.83 \mathrm{e}-1$ & - & $1.03 \mathrm{e}+0$ & - \\
\hline $2^{-2}$ & $4.31 \mathrm{e}-2$ & 2.07 & $2.42 \mathrm{e}-1$ & 2.06 \\
\hline $2^{-3}$ & $1.03 \mathrm{e}-2$ & 2.01 & $5.78 \mathrm{e}-2$ & 2.01 \\
\hline $2^{-4}$ & $2.54 \mathrm{e}-3$ & 2.00 & $1.43 \mathrm{e}-2$ & 2.00 \\
\hline $2^{-5}$ & $6.35 \mathrm{e}-4$ & 2.00 & $3.57 \mathrm{e}-3$ & 2.00 \\
\hline
\end{tabular}

Table 9: Errors and rates with Gauss I time-stepping method and $\mathrm{RT}_{2}-\mathrm{NIPG}_{3}$.

\begin{tabular}{|c|c|c|c|c|}
\hline \multicolumn{5}{|c|}{ Pressure and velocity } \\
\hline$\Delta t$ & $\left\|p-p_{h}\right\|_{L^{2}(\Omega)}$ & Cvg. rate & $\left\|\mathbf{u}-\mathbf{u}_{h}\right\|_{L^{2}(\Omega)}$ & Cvg. rate \\
\hline $2^{-1}$ & $1.12 \mathrm{e}-1$ & - & $9.73 \mathrm{e}-5$ & - \\
\hline $2^{-2}$ & $1.33 \mathrm{e}-2$ & 3.30 & $1.23 \mathrm{e}-5$ & 3.30 \\
\hline $2^{-3}$ & $1.35 \mathrm{e}-3$ & 3.15 & $1.25 \mathrm{e}-6$ & 3.16 \\
\hline $2^{-4}$ & $1.52 \mathrm{e}-4$ & 2.92 & $1.39 \mathrm{e}-7$ & 3.00 \\
\hline $2^{-5}$ & $2.00 \mathrm{e}-5$ & 2.13 & $1.75 \mathrm{e}-8$ & 1.55 \\
\hline \hline \multicolumn{5}{|c|}{ Concentration } \\
\hline$\Delta t$ & $\left\|c-c_{h}\right\|_{L^{2}(\Omega)}$ & Cvg. rate & $\left\|\nabla c-\nabla c_{h}\right\|_{L^{2}\left(\mathcal{E}_{h}\right)}$ & Cvg. rate \\
\hline $2^{-1}$ & $2.65 \mathrm{e}-1$ & - & $1.49 \mathrm{e}+0$ & - \\
\hline $2^{-2}$ & $3.48 \mathrm{e}-2$ & 3.29 & $1.95 \mathrm{e}-1$ & 3.29 \\
\hline $2^{-3}$ & $3.56 \mathrm{e}-3$ & 3.16 & $2.00 \mathrm{e}-2$ & 3.15 \\
\hline $2^{-4}$ & $3.98 \mathrm{e}-4$ & 3.07 & $2.24 \mathrm{e}-3$ & 3.02 \\
\hline $2^{-5}$ & $4.72 \mathrm{e}-5$ & 2.98 & $2.76 \mathrm{e}-4$ & 2.45 \\
\hline
\end{tabular}

Table 10: Errors and rates with Radau II time-stepping method and $\mathrm{RT}_{2}-\mathrm{NIPG}_{3}$. 

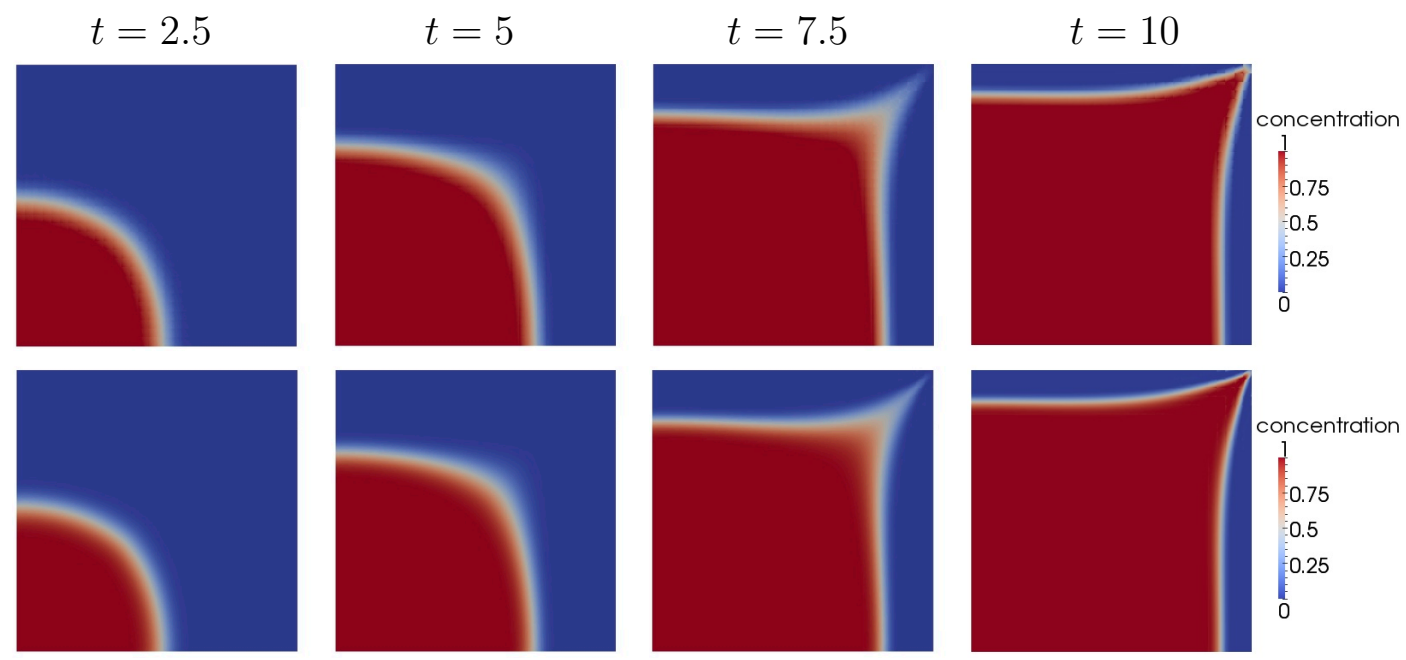

Figure 1: Simulations of the fluid concentration with Implicit Euler in time: the first row corresponds to $\mathrm{RT}_{1}-\mathrm{NIPG}_{1}$ and the second row to $\mathrm{RT}_{2}-\mathrm{NIPG}_{2}$. Mesh size is $1 / 64$.
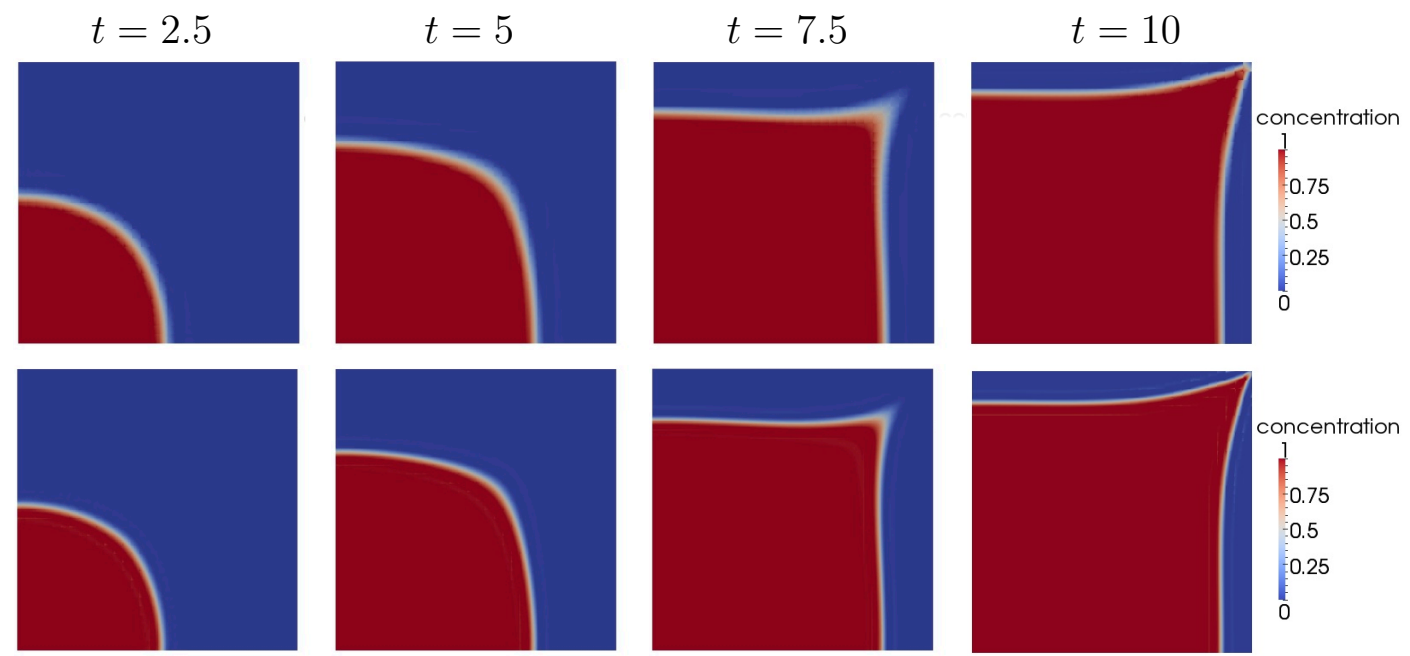

Figure 2: Simulations of the fluid concentration with Gauss I method in time: the first row corresponds to $\mathrm{RT}_{1}-\mathrm{NIPG}_{1}$ and the second row to $\mathrm{RT}_{2}-\mathrm{NIPG}_{2}$. Mesh size is $1 / 64$. 

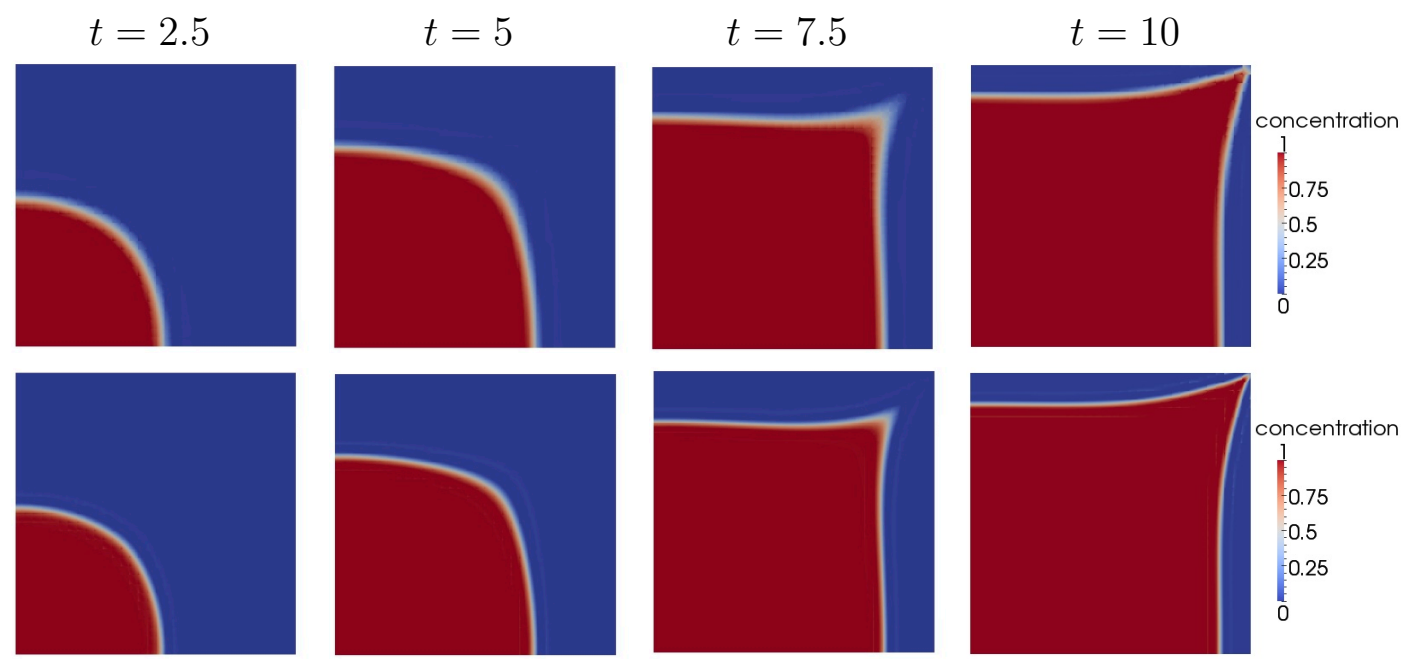

Figure 3: Simulations of the fluid concentration with Radau II method in time: the first row corresponds to $\mathrm{RT}_{1}-\mathrm{NIPG}_{1}$ and the second row to $\mathrm{RT}_{2}-\mathrm{NIPG}_{2}$. Mesh size is $1 / 64$.
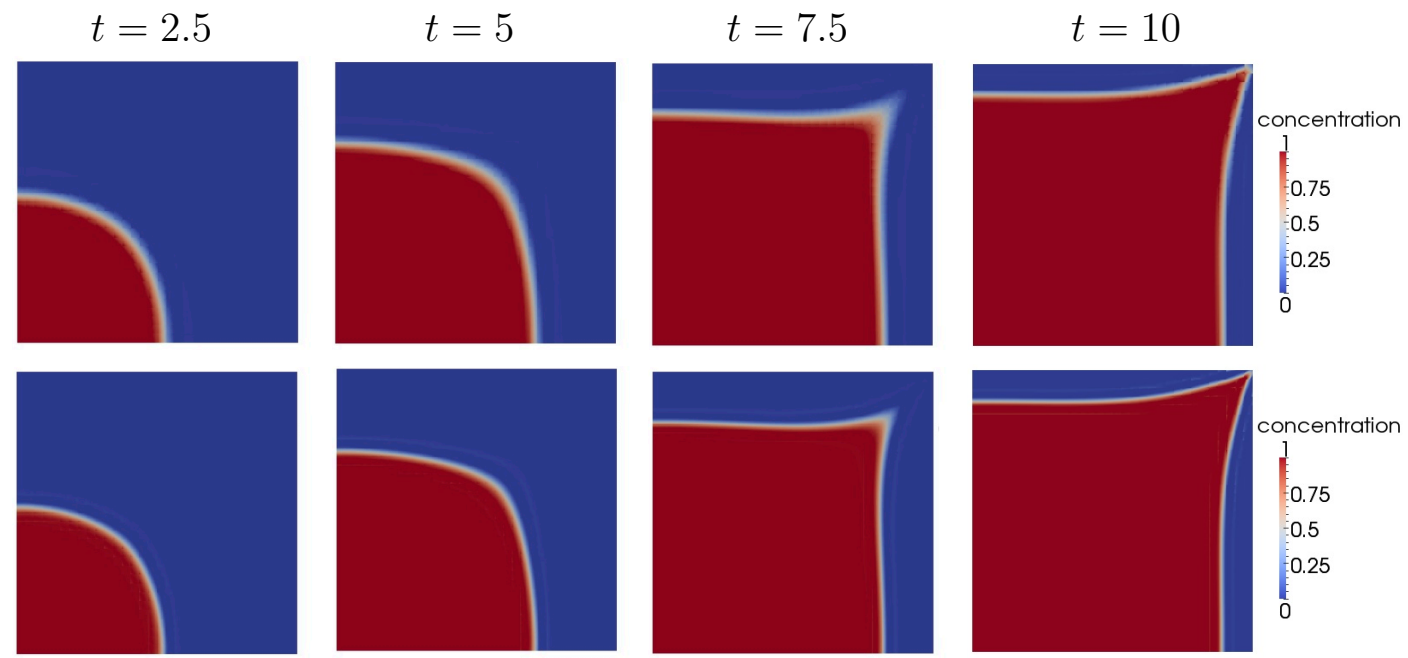

Figure 4: Simulations of the fluid concentration with Lobatto III method in time: the first row corresponds to $\mathrm{RT}_{1}-\mathrm{NIPG}_{1}$ and the second row to $\mathrm{RT}_{2}-\mathrm{NIPG}_{2}$. Mesh size is $1 / 64$. 


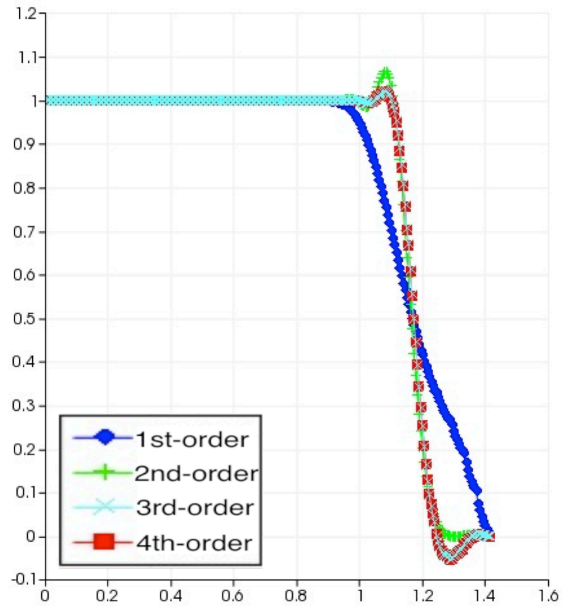

Figure 5: Profiles of concentration along the diagonal.

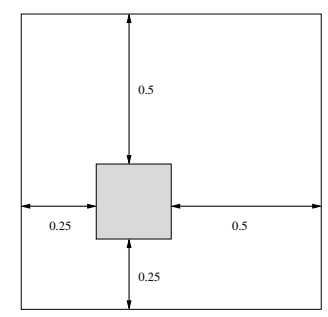

Figure 6: Example of medium with square inclusion. 

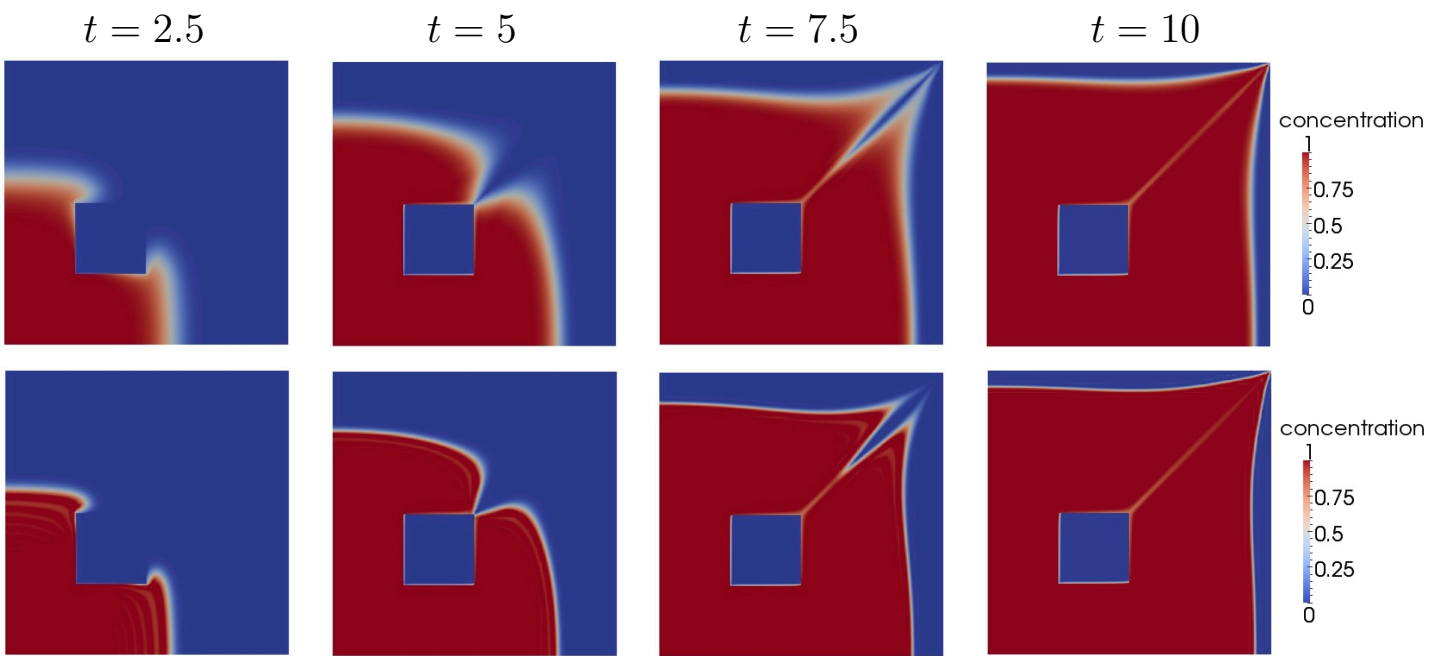

Figure 7: Simulations of the fluid concentration with $\mathrm{RT}_{2}-\mathrm{NIPG}_{2}$ : the first row corresponds to first order in time and the second row to second order in time. Mesh size is $1 / 64$.
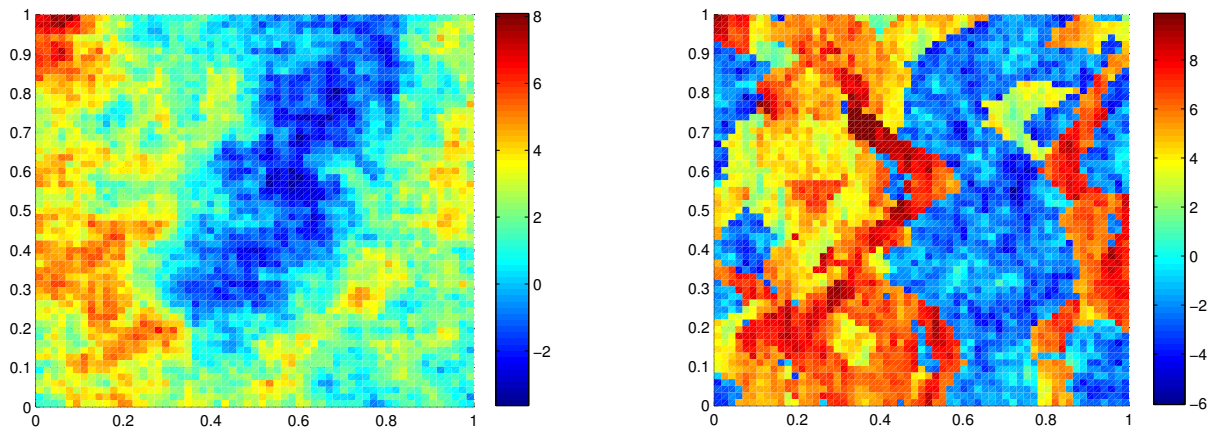

Figure 8: Two heterogeneous permeability fields from the SPE10 benchmark problem: Tarbert formation (left) and Upper Ness formation (right). Spatial resolution is $64 \times 64$. 

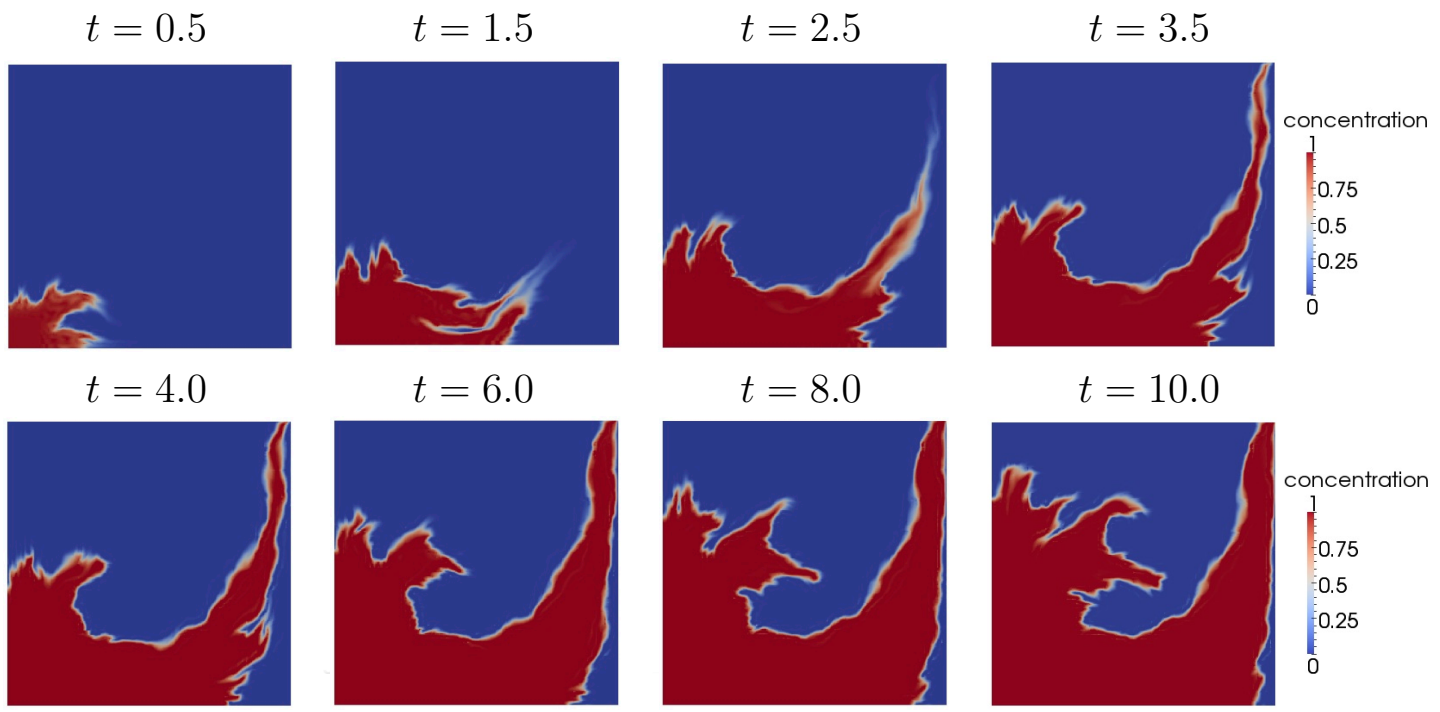

Figure 9: Simulations of the fluid concentration with $\mathrm{RT}_{2}-\mathrm{NIPG}_{2}$ and Gauss I method for the Tarbet formation. Mesh size is $1 / 64$.
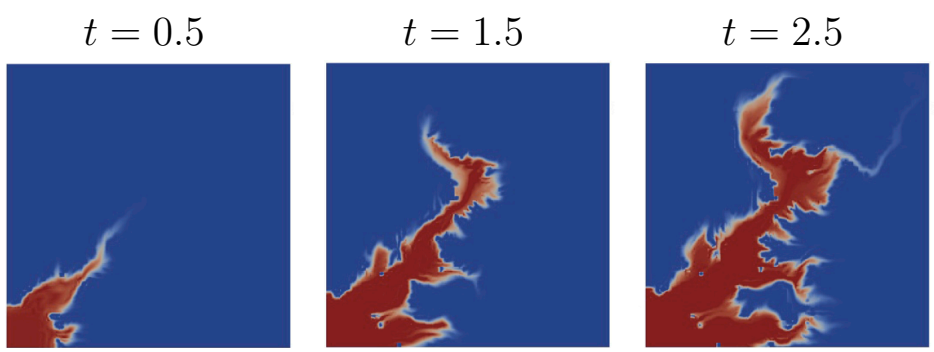

$$
t=4.0
$$
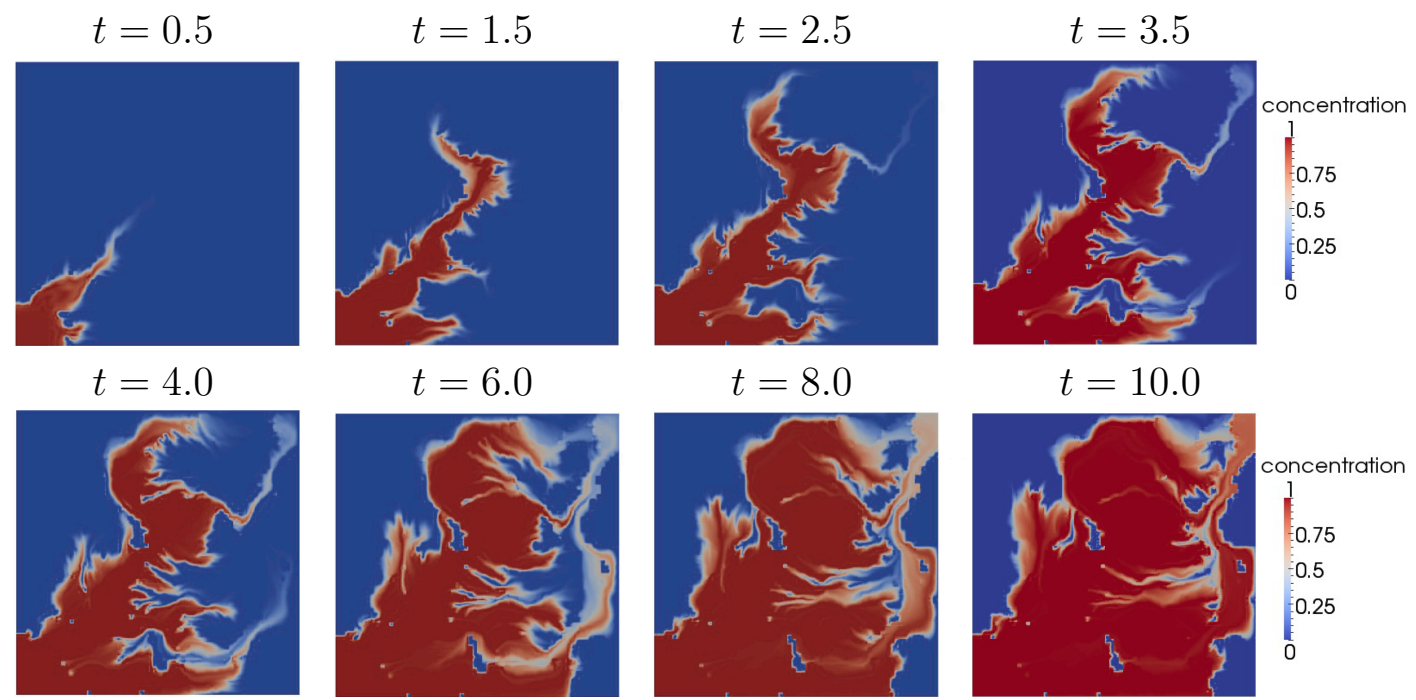

Figure 10: Simulations of the fluid concentration with $\mathrm{RT}_{2}-\mathrm{NIPG}_{2}$ and Gauss I method for the Upper-Ness formation. Mesh size is $1 / 64$. 